\title{
Diagnosis and treatment of cervical incompetence combined with intrauterine adhesions
}

\author{
Waixing Li ${ }^{\#}$, Yueran $\mathrm{Li}^{\#}$, Xingping Zhao, Chunxia Cheng, Arvind Burjoo, Yimin Yang, Dabao Xu \\ Department of Obstetrics and Gynecology, The Third Xiangya Hospital of Central South University, Changsha 410013, China \\ Contributions: (I) Conception and design: D Xu, Y Yang; (II) Administrative support: D Xu; (III) Provision of study materials: Y Li, X Zhao, C Cheng; \\ (IV) Collection and assembly of data: Y Li, W Li; (V) Data analysis and interpretation: Y Li, W Li; (VI) Manuscript writing: All authors; (VII) Final \\ approval of manuscript: All authors. \\ "These authors contributed equally to this work. \\ Correspondence to: Dabao Xu; Yimin Yang. Department of Obstetrics and Gynaecology, The Third Xiangya Hospital of Central South University, 138 \\ Tongzipo Road, Changsha 410013, China. Email: forxudabao@126.com; 492503562@qq.com.
}

Background: Cervical insufficiency (CI) with concomitant intrauterine adhesions (IUAs) is a common clinical phenomenon among CI patients. But there are neither published reports regarding the difference in diagnosis and treatment of such patients compared to those with CI only, nor any report about their prognosis. This study aimed to preliminary the alteration in diagnostic and curative aspects of these patients, so as to provide a certain reference for the clinical management of such conditions.

Methods: Ten patients with CI combined with moderate to severe IUAs were diagnosed, treated and followed up at the Third Xiangya Hospital of Central South University from September 2017 to August 2019, their medical records and the pregnancy outcomes were retrospectively analyzed.

Results: All 10 patients had a previous history of typical painless cervical dilatation during the second trimester. All patients were moderate to severer IUAs, and the mean AFS score of IUAs was 9.80 \pm 1.08 (range, 8 to 12). Preoperatively, in 6 patients, the No. 7 Hegar dilator was able to pass through the internal cervical os before surgery without resistance. In the other 4 patients, the Hegar dilator could not be inserted before surgery due to the adhesions of the cervical canal and the lower uterine segment; the diagnoses of these patients were further confirmed at 3 months after hysteroscopic adhesiolysis (HA) when the No. 7 Hegar dilator was able to pass through the internal cervical os without resistance. There were 9 patients underwent pre-pregnancy laparoscopic cervical cerclage after HA. The remaining 1 patient exceptionally underwent laparoscopic cervical cerclage prior to HA, as the cervix was too loose to retain and be treated with an intrauterine device (IUD) or distended Foley's catheter balloon; which essentially prevent postoperative adhesion reformation. The patients were followed-up for 3 months to 2 years. The pregnancy rate was $60 \%$, and the live birth rate was $100 \%$.

Conclusions: In patients with CI and concomitant cervical or lower uterine segment IUAs, it is necessary to separate the adhesion prior to evaluating the cervical competency with the No. 7 Hegar dilator, to confirm the diagnosis. However, when the cervix is too loose, laparoscopic cervical cerclage is exceptionally carried out first and then IUAs is treated. Pre-pregnancy laparoscopic cervical cerclage has a good prognosis in patients with CI complicated by moderate to severe IUAs.

Keywords: Cervical incompetence (CI); intrauterine adhesions (IUAs); laparoscopic cervical cerclage; diagnosis; live birth rate

Submitted Nov 13, 2019. Accepted for publication Dec 26, 2019.

doi: $10.21037 /$ atm.2019.12.148

View this article at: http://dx.doi.org/10.21037/atm.2019.12.148 


\section{Introduction}

Cervical incompetence (CI) is one of the main causes of premature birth or miscarriage in the second trimester. According to statistics, $8 \%$ of miscarriages in the second trimester and premature births are due to CI (1). If a patient has a history of typical painless cervical dilation during the second trimester, and a No. 7 Hegar dilator can pass through the internal cervical os without resistance during the nonpregnancy period, the diagnosis of CI can be confirmed (2). The surgical treatment of CI is cervical cerclage which refers to a variety of procedures that use sutures or synthetic tape to reinforce the cervix. Cervical cerclage can be done through the vagina (transvaginal cervical cerclage) or, less commonly, through the abdomen (transabdominal or laparoscopic cervical cerclage). The prophylactic cervical cerclage should preferably be performed before or in the early period of pregnancy. Both approaches have their own advantages and disadvantages. Currently, the most commonly used method is the transvaginal approach, but upon its failure, laparoscopic cervical cerclage is recommended.

Intrauterine adhesions (IUAs) encompass adhesions and atresia of the uterine cavity and cervical canal secondary to damage of the endometrial basal layer caused by intrauterine operations, infections, and other factors (3). $\mathrm{CI}$ is the main cause of recurrent abortions which usually resort to curettage. In turn, curettage is the most common etiological factor for IUAs, and that explains the coexistence of CI \& IUAs. At present, hysteroscopy is the gold standard for the diagnosis and treatment of IUAs. There are some differences in the diagnosis and treatment between the CI combined with IUAs and only CI. There are two main problems. On the one hand, for patients with adhesions of the cervical canal or the lower uterine segment, a Hegar dilatator is often unable to enter the uterine cavity or it enters with difficulty, which can easily lead to a missed diagnosis of CI and increase the risk of uterine perforation. On the other hand, when the cervix is too loose, more difficulties are encountered in HA and in the postoperative prevention of adhesion reformation. Currently, studies on the diagnosis, treatment, and prognosis of such patients have not been published. This study preliminary the diagnosis and treatment modalities of such patients and followed up the pregnancy outcomes after treatment, so as to provide a certain reference for future clinical treatment of such conditions.

\section{Methods}

\section{General information}

The clinical information of 10 patients with CI combined with moderate to severe IUAs who were treated at the Third Xiangya Hospital of the Central South University from September 2017 to August 2019 were retrospectively analyzed. Among them, 9 patients underwent HA and IUD and intrauterine balloon placement first and upon restoration of a normal uterine cavity morphology, prepregnancy laparoscopic cervical cerclage was performed. After counselling, the remaining 1 patient underwent prepregnancy laparoscopic cervical cerclage prior to HA as the use of IUD and intrauterine balloon (to prevent postoperative adhesion) were hampered by an excessively loose cervix. The mean patient age was $31.20 \pm 2.57$ years (range, 28 to 36 years). Before the laparoscopic cervical cerclage, the mean number of pregnancies were $3.70 \pm 1.80$ ( 2 to 8 ) and the mean number of adverse pregnancies were $1.80 \pm 0.63$ ( 1 to 3 ). Four patients had a history of previous cervical surgery or injury. Among them, 2 patients underwent transvaginal cervical cerclage during the second trimester, but premature delivery or miscarriage during the second trimester still occurred, 1 patient had a previous cervical loop electrosurgical excision procedure (LEEP), and 1 experienced cervical laceration during a previous delivery.

\section{Inclusion criteria}

Patients diagnosed with moderate to severe IUAs combined with CI and who had a fertility desire were included.

(I) The American Fertility Society (AFS) scoring system for IUAs (4): a score of 1 to 4 points is classified as mild, 5 to 8 as moderate, and 9 to 12 as severe.

(II) Diagnostic criteria of CI (2): (i) multiple spontaneous miscarriages during the second trimester, (ii) loose cervical canal during nonpregnancy, as reflected by the No. 7 Hegar dilator passing through without resistance. CI is diagnosed if either (or both) of the above 2 criteria is satisfied.

\section{Exclusion criteria}

Acute inflammation of the internal genitalia or systemic acute inflammation, severe pelvic adhesion that is not suitable for laparoscopic surgery, age older than 40 years, 


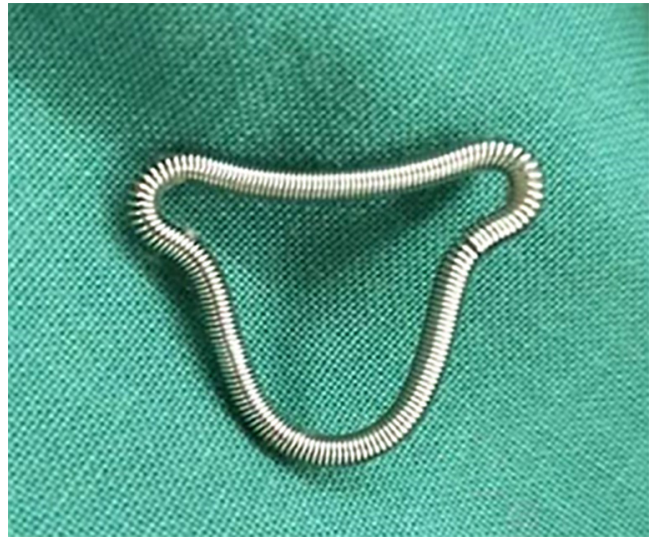

Figure 1 Uterine-shaped loop IUD. IUD, intrauterine device.

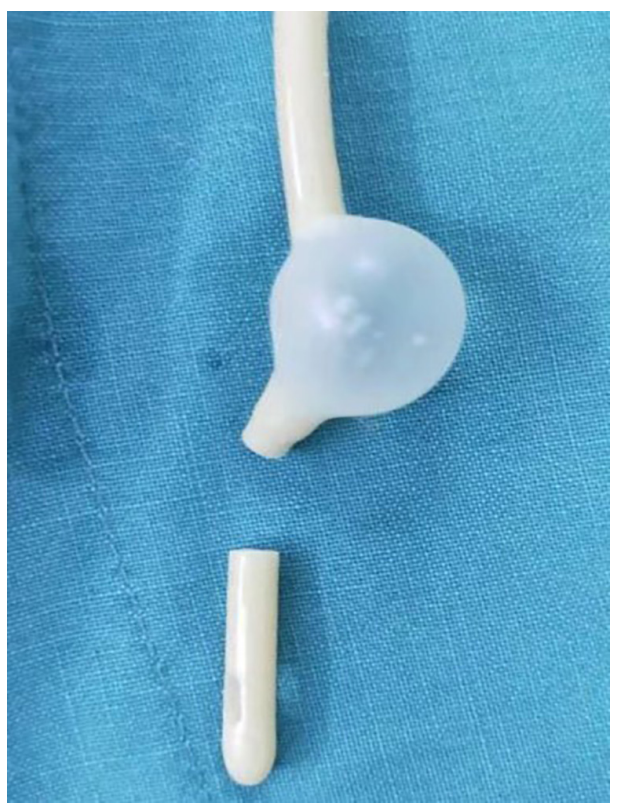

Figure 2 Foley's catheter balloon, with the top catheter portion beyond the balloon removed.

decreased ovarian reserve function [follicle stimulating hormone $(\mathrm{FSH}) \geq 10 \mathrm{IU} / \mathrm{L}]$, and fetal abnormalities or other adverse reproductive outcomes history. Patients with the above conditions were advised to perform cerclage during pregnancy after screening at 14 weeks of pregnancy.

\section{Surgical methods and postoperative management}

Hysteroscopic adhesiolysis (HA): Bettocchi hysteroscope with a $5.4 \mathrm{~mm}$ outer diameter and a 5-Fr working channel was used to enter the cervical canal in order to prevent a misdiagnosis of CI caused by IUAs. After a confirmed diagnosis of IUAs, HA was carried out using 5-Fr double action forceps and single action scissors $(5,6)$. After adhesiolysis a suitable sized IUD (Figure 1) was placed into the intrauterine cavity and whether the size and position of the IUD was suitable was verified right after its placement via hysteroscopy. Estradiol valerate at $3 \mathrm{mg} / \mathrm{Bid}$ was administered starting on the 5 th day of the menstrual period for 21 consecutive days, and progesterone at $200 \mathrm{mg}$ q.n. was added starting on the 16th day of the 21 days for 6 consecutive days. Following up hysteroscopy was carried out 1 month and 4 months after the initial HA. If the follow-up hysteroscopy showed adhesions, they were separated again. The cervical cerclage was performed until the AFS score was less than 5. The IUD of one patient moved down to the cervical canal and the balloon (Figure 2) fell off within one day after HA due to the very loose cervix. The patient was reviewed one month after initial HA. Hysteroscopy showed a recurrent IUAs with an AFS score of 7 and cervical cerclage was performed followed by HA at the same time.

Laparoscopic cervical cerclage: the vesicouterine peritoneum is opened using the monopolar L-hook electrode and dissected off the lower uterine segment, exposing the uterine vessels anteriorly on both sides. A 5-mm nonabsorbable Mersilene polyester suture (Johnson and Johnson company), with adjacent straightened blunt needles was introduced through the laparoscopic port into the abdominal cavity. The stitch was placed by passing each needle between the uterine vessels and the uterine isthmus from anterior to posterior, at the level of the internal cervical os bilaterally. The needles were then cut off and removed, and the Mersilene suture was then tied tightly around the cervix with four knots using intracorporeal knot tying. The ends of the stitch were trimmed. No suture penetration was detected by hysteroscopy. The cervix accommodated up to the No. 6 Hegar dilator without resistance but cannot through the No. 8 Hegar dilator.

\section{Evaluation indexes of the treatment effect}

Pregnancy status after treatment: The pregnancy rate, miscarriage rate, live birth rate, incidence of preterm premature rupture of membranes, full-term delivery rate, blood loss during cesarean section, and placental adhesions were assessed. 
Table 1 Pregnancy and pregnancy outcomes

\begin{tabular}{lc}
\hline Variables & Outcomes \\
\hline Postoperative pregnancy (pregnancy rate) & $6(60 \%)$ \\
Live birth rate & $100 \%$ \\
Gestation week at delivery (weeks) & $37.2 \pm 1.17$ \\
$<37$ & $1(16.7 \%)$ \\
$\geq 37$ & $5(83.3 \%)$ \\
Miscarriage rate & 0 \\
Premature rupture of membranes & 0 \\
Placental adhesion & $2(33.3 \%)$ \\
Blood loss during cesarean section $(\mathrm{mL})$ & $283 \pm 263$ \\
\hline
\end{tabular}

\section{Results}

Diagnosis and treatment of CI: all 10 patients had a previous history of typical painless cervical dilation during the second trimester before surgery, and in 6 patients, the No. 7 Hegar dilator could pass through the internal cervical os before surgery without resistance. In the other 4 patients, the cervical dilator could not be inserted before surgery due to adhesions of the cervical canal and the lower uterine segment; the diagnoses of these patients were further confirmed 3 months after the HA when the No. 7 Hegar dilator was able to pass through the internal cervical os without resistance. There were 9 patients who underwent pre-pregnancy laparoscopic cervical cerclage after HA. The remaining 1 patient underwent laparoscopic cervical cerclage prior second HA, as the cervix was too loose to retain and be treated with an IUD or distended Foley's catheter balloon (Figures 1,2) which essentially prevent postoperative adhesion reformation. Ten patients underwent laparoscopic cervical cerclage with an intraoperative blood loss of 10-50 mL, with an average blood loss of $27 \pm$ $16.16 \mathrm{~mL}$.

Diagnosis and treatment of IUAs: all 10 patients were diagnosed with IUAs by hysteroscopy. The mean AFS score of the initial IUAs was $9.80 \pm 1.08$ (range, 8 to 12 ) and the mean number of HA was $2.8 \pm 1.03$ (2 to 5 ). At the end of treatment of IUAs, the mean AFS score was $2.70 \pm 1.00$.

\section{Pregnancy and pregnancy outcomes after the treatment of CI concomitant with IUAs (see Table 1)}

Pregnancy: in all, 10 patients were followed-up. Six patients were pregnant [5 with natural conception and 1 who underwent in vitro fertilization/embryo transfer (IVF/ET)]. The occurrence time of the pregnancy was 1 to 2 months after treatment, with an average of $1.2 \pm 0.4$ months. Four patients were not pregnant ( 2 patients each actively prepared for pregnancy for 3 and 9 months after surgery, but were still not pregnant. The other 2 patients underwent IVF-ET 1 month after surgery, but both had failed conceptions due to a lack of "implantation of embryo").

Pregnancy outcomes: the mean delivery gestational age was $37.2 \pm 1.17$ weeks (range, 35 to 38 ). One patient underwent cesarean section due to preterm labor at 35 weeks of pregnancy without premature rupture of membranes; the remaining 5 patients underwent elective cesarean sections at 37 to 38 weeks. six patients who underwent cesarean section experienced no serious surgical complications. The intraoperative blood loss was $100-800 \mathrm{~mL}$, with an average of $283 \pm 263 \mathrm{~mL}$.

Four patients had intact placentas and membranes during cesarean delivery. The placental adhesions were identified during cesarean section in 2 patients, and manual detachment of the placenta was performed.

\section{Discussion}

$\mathrm{CI}$ is defined as the inability of the uterine cervix to retain a pregnancy in the second trimester in the absence of clinical contractions, labor, or both (2). IUAs refers to the disease caused by trauma or inflammation of the endometrium. It is most often secondary to endometrial basal layer injury, such as curettage, cesarean section, abdominal myomectomy and/or hysteroscopic myomectomy, polypectomy or incision of the uterine septum $(7,8)$. As CI patients usually undergo multiple uterine operations, they are prone to develop IUAs and this explains the co-existence of these two conditions. Accurate diagnosis while avoiding missed diagnosis is a necessary first step in managing these patients and eventually improving their prognosis.

According to the American College of Obstetricians and Gynecologists (ACOG) guidelines (2), the diagnostic criteria for simple CI are based on 3 aspects: medical history, ultrasound indicators, and a tentative diagnosis during the nonpregnancy period. Among them, the medical history is the most important for the diagnosis of $\mathrm{CI}$, and a history of miscarriage in the second trimester or premature birth caused by repeated painless cervical dilatation is the direct basis for the diagnosis of CI. At the 
same time, a cervical canal width $>0.6 \mathrm{~cm}$ at the internal cervical os under ultrasound examination can also assist in the diagnosis. Other diagnostic modalities of CI include: hysterosalpingography (HSG) and imaging of balloon traction on the cervix radiographically, assessment of the patulous cervix with Hegar or Pratt dilators, balloon elastance test, and graduated cervical dilators which are used to calculate the cervical resistance index based on the functional anatomy of the internal os in the non-pregnant state. In this study, CI was diagnosed upon easy passage of the No. 7 Hegar dilator through the intracervical canal.

The diagnosis of CI combined with IUAs should be carried out with diligence as the inability or difficulty in introducing the Hegar dilator may lead to missed diagnosis, creation of false passage and even cause uterine perforation. we were unable to introduce the Hegar dilator into the cervical canal in 4 of them due to the adhesion. However, after HA, diagnosis of CI was confirmed as the No. 7 Hegar dilator accessed the cervical canal without any resistance. Therefore, for patients with CI, complicated with moderate to severe IUAs, especially of the cervix and lower uterine segment, it is recommended to insert the cervical dilator again after the uterine cavity morphology returns to normal or 3 months after the initial HA; as it is more safe and reliable postoperatively and it effectively avoids missed diagnosis.

Cervical cerclage is the mainstay of surgical treatment for $\mathrm{CI}$ and the approaches include transvaginal and transabdominal cervical cerclage (9). The most effective method is laparoscopic cervical cerclage through transabdominal approach. For patients with simple CI, transvaginal cervical cerclage is currently the most commonly performed procedure. However, studies show that $11-53 \%$ of patients who underwent transvaginal cervical cerclage still experienced miscarriage, and patients who had failed transvaginal cervical cerclage underwent transabdominal cerclage and achieved a live birth rate of more than $90 \%$ (10-13). This finding is due to the relatively low cerclage position in transvaginal cervical cerclage. Moreover, transvaginal cerclage is more likely to fail for patients who underwent LEEP or with the short cervix. The laparoscopic cervical cerclage site is close to the internal cervical os, which can greatly reduce the possibility of surgical failure caused by low cerclage position (10). Compared with transvaginal cervical cerclage, laparoscopic cervical cerclage is more complicated, the cerclage band can only be removed by cesarean section, and there is an increased risk of pelvic adhesion and bladder injury. But for the special population of patients with IUAs combined with CI, the benefits of laparoscopic cervical cerclage outweigh its drawbacks as it is primordial in ensuring increased live birth rate. Patients with IUAs are prone to miscarriages, and those who also have CI are more likely to have miscarriages during the second and third trimester or premature labor when compared cervical cerclage transvaginally to laparoscopically. If curettage is performed again due to miscarriage, damage to the uterine cavity may lead to irreversible chances to reproduction as the uterine cavity and the endometrium had already been severely damaged previously. Therefore, for patients with moderate to severe IUAs combined with CI, we recommend pre-pregnancy laparoscopic cervical cerclage to maximally reduce the risk of miscarriage or premature birth. Many studies have demonstrated that compared with emergency cervical cerclage treatment, elective cervical cerclage treatment for CI can significantly prolong the gestation weeks, shorten the hospitalization time, and improve neonatal prognosis (14). In addition, pre-pregnancy laparoscopic cervical cerclage not only can prevent miscarriage but also won't limit conception or assisted reproductive technology (13). Of the 10 patients we treated, 6 (60\%) were pregnant 1-2 months after surgery. Among them, one patient had a previous cervical LEEP, and 1 patient had 2 previous failed transvaginal cervical cerclages. The laparoscopic cervical cerclage is simple and minimally invasive. In our study, the average amount of blood loss during pre-pregnancy laparoscopic cervical cerclage surgery was very small $(27 \pm 16.16 \mathrm{~mL})$ and there was no surgical complication. After the treatment of CI and IUAs, the average gestational age was 37 weeks, and the fullterm delivery rate was $83 \%$. Only 1 patient delivered at 35 weeks by cesarean section due to preterm labor. The prognosis of the newborns was good. No patient had midterm miscarriage, premature rupture of membranes, chorioamnionitis, etc. One-third of the patients (2 patients) had placental adhesions, and thus manual placental detachment was performed with an average intraoperative blood loss of $283 \pm 263 \mathrm{~mL}$. In our study, pre-pregnancy laparoscopic cervical cerclage for moderate to severe IUAs concomitant with $\mathrm{CI}$ has a relatively good prognosis.

The order of treatment of CI and IUAs should be decided according to the specific circumstances. Generally, it is recommended to treat IUAs first and then to treat CI. The reasons are as follows: (I) the treatment of IUAs does not surely have a good prognosis, especially for a totally destroyed uterine cavity; (II) to avoid cerclage band 
damage caused by multiple intrauterine operations; (III) for patients with adhesions of the cervical canal and lower uterine segment, the diagnosis needs to be confirmed after HA. However, for special cases, such as excessive looseness of the inner orifice of the cervix, whereby IUD or balloon cannot be used in order to prevent postoperative adhesion reformation, it is necessary to counsel and advise the patient about her underlying condition. After obtaining the understanding and consent of the patient, the laparoscopic cervical cerclage can be exceptionally performed first and then treat IUAs. Among the 10 patients treated, 9 patients underwent pre-pregnancy laparoscopic cervical cerclage when the IUAs was cured or recovered to the point where the AFS score was mild enough to consider pregnancy. And the remaining 1 patient underwent laparoscopic cervical cerclage before the final HA, because the cervix was too loose to be treated with intrauterine device (IUD) or balloon to prevent postoperative adhesion reformation.

In summary, patients with CI complicated with IUAs of the cervix or lower uterine segment, it is necessary to separate the adhesion first and then evaluate the cervix with the No. 7 Hegar dilator to confirm the diagnosis. However, when the cervix is too loose, laparoscopic cervical cerclage is exceptionally carried out first and then IUAs is treated. Patients with CI complicated with moderate to severe IUAs who underwent pre-pregnancy laparoscopic cervical cerclage had a relatively good prognosis.

\section{Acknowledgments}

Funding: We are grateful for the support of the Hunan Science and Technology Department (Grant No.2018SK2102) and the Changsha Science and Technology Bureau (Grant No. kq1901124) of China.

\section{Footnotes}

Conflicts of Interest: The authors have no conflicts of interest to declare.

Ethical Statement: The authors are accountable for all aspects of the work in ensuring that questions related to the accuracy or integrity of any part of the work are appropriately investigated and resolved. This study was approved by the Ethics Committee of the Third Xiangya Hospital of Central South University (Project number: 2019-S535).

\section{References}

1. Alfirevic Z, Stampalija T, Medley N. Cervical stitch (cerclage) for preventing preterm birth in singleton pregnancy. Cochrane Database Syst Rev 2017;6:CD008991.

2. American College of Obstetricians and Gynecologists. ACOG Practice Bulletin No.142: Cerclage for the management of cervical insufficiency. Obstet Gynecol 2014;123:372-9.

3. Zhang L, Wang M, Zhang Q, et al. Estrogen therapy before hysteroscopic adhesiolysis improves the fertility outcome in patients with intrauterine adhesions. Arch Gynecol Obstet 2019;300:933-9.

4. The American Fertility Society classifications of adnexal adhesions, distal tubal occlusion, tubal occlusion secondary to tubal ligation, tubal pregnancies, müllerian anomalies and intrauterine adhesions. Fertil Steril 1988;49:944-55.

5. Zhang A, Jamail G, Xue M, et al. Hysteroscopic Intrauterine Adhesiolysis Using the "Ploughing" Technique With Cold Scissors. J Minim Invasive Gynecol 2015;22:934-5.

6. Huang H, Cheng C, Johnson G, et al. Hysteroscopic Intrauterine Adhesiolysis Using a Blunt Spreading Dissection Technique with a Double Action Forceps. J Minim Invasive Gynecol 2018;25:583-4.

7. Warembourg S, Huberlant S, Garric X, et al. Prevention and treatment of intra-uterine synechiae: review of the literature. J Gynecol Obstet Biol Reprod (Paris) 2015;44:366-79.

8. Taskin O, Sadik S, Onoglu A, et al. Role of endometrial suppression on the frequency of intrauterine adhesions after resectoscopic surgery. J Am Assoc Gynecol Laparosc 2000;7:351-4.

9. Brown R, Gagnon R, Delisle MF. No. 373-Cervical Insufficiency and Cervical Cerclage. J Obstet Gynaecol Can 2019;41:233-47.

10. Huang X, Ma N, Li TC, et al. Simplified laparoscopic cervical cerclage after failure of vaginal suture: technique and results of a consecutive series of 100 cases. Eur J Obstet Gynecol Reprod Biol 2016;201:146-50.

11. Zaveri V, Aghajafari F, Amankwah K, et al. Abdominal versus vaginal cerclage after a failed transvaginal cerclage: a systematic review. Am J Obstet Gynecol 2002;187:868-72.

12. Novy MJ, Gupta A, Wothe DD, et al. Cervical cerclage in the second trimester of pregnancy: a historical 
cohort study. Am J Obstet Gynecol 2001;184:1447-54; discussion 1454-6.

13. Sumners JE, Kuper SG, Foster TL. Transabdominal Cerclage. Clin Obstet Gynecol 2016;59:295-301.

Cite this article as: $\mathrm{Li} \mathrm{W,} \mathrm{Li} \mathrm{Y,} \mathrm{Zhao} \mathrm{X,} \mathrm{Cheng} \mathrm{C,} \mathrm{Burjoo} \mathrm{A,}$ Yang Y, Xu D. Diagnosis and treatment of cervical incompetence combined with intrauterine adhesions. Ann Transl Med 2020;8(4):54. doi: 10.21037/atm.2019.12.148
14. Krispin E, Danieli-Gruber S, Hadar E, et al. Primary, secondary, and tertiary preventions of preterm birth with cervical cerclage. Arch Gynecol Obstet 2019;300:305-12. 